\title{
The Benefits of Mindfulness Meditation on Trait Mindfulness, Perceived Stress, Cortisol, and C-Reactive Protein in Nursing Students: A Randomized Controlled Trial
}

\author{
Hossam N Alhawatmeh (D) \\ Mohammad Rababa (D) \\ Mahmoud Alfaqih (D) ${ }^{2}$ \\ Raya Albataineh ${ }^{2}$ \\ Issa Hweidi (D) \\ Awwad Abu Awwad' \\ 'Jordan University of Science of \\ Technology, Faculty of Nursing, Irbid, \\ Jordan; ${ }^{2}$ Jordan University of Science of \\ Technology, Faculty of Medicine, Irbid, \\ Jordan
}

\begin{abstract}
Purpose: Mindfulness meditation was used to reduce stress and its responses such as cortisol and C-reactive protein (CRP) among healthy and ill individuals in various cultures, but its effect has not yet been studied among nursing students, experiencing tremendous stress. The objective of this study was to examine the effects of mindfulness meditation on trait mindfulness, perceived stress, serum cortisol, and serum C-reactive proteins (CRP) in nursing students.
\end{abstract}

Patients and Methods: Using a two-arm, randomized, parallel study (conducted in a large university in Jordan, 108 nursing students were randomly assigned to experimental group receiving five 30-minute weekly sessions of mindfulness meditation and control group sitting quiet during the experimental sessions. Trait mindfulness, perceived stress, serum cortisol, and CRP were measured at baseline and end of the intervention.

Results: Using one-way MANOVA and post-hoc comparisons, the results showed that mindfulness meditation was significantly effective in decreasing serum cortisol levels and perceived stress. The mindfulness meditation also decreased CRP and increased trait mindfulness although the results did not reach statistically significant levels.

Conclusion: These findings underscore the need for serious consideration of mindfulness meditation in nursing colleges to improve stress and raise immunity in this vulnerable population.

Trial Registration: Mindfulness Meditation for Nursing Students: clinicaltrials.gov, identifier: NCT05099224.

Keywords: mindfulness, meditation, nursing students, stress, cortisol, C-reactive proteins

\section{Introduction}

Stress has been acknowledged as an important health issue in nursing education worldwide. ${ }^{1}$ Nursing students have been found to have high levels of chronic stress, sometimes higher than the levels reported by students from other health professions. $^{2,3}$ Previous studies have shown that repeated exposure to stress events can negatively impact the body. Specifically, the immune system can be impacted by the activation of the hypothalamic-pituitary-adrenal (HPA) system (eg, cortisol). ${ }^{4,5}$

Upon perception of a stressful situation or event, the HPA axis is activated by the amygdala which signals the hypothalamus to secrete corticotropin-releasing hormone
Correspondence: Hossam N Alhawatmeh Tel +962797413244

Fax +962-2-7201065

Email hnhawatmeh@just.edu.jo 
$(\mathrm{CRH})$. CRH then stimulates the release of adrenocorticotropic hormone (ACTH) from the anterior areas of the pituitary gland. ${ }^{5}$ ACTH stimulates the adrenal cortex to secrete cortisol that increases systemically 15 minutes after the stress perception and remains elevated for several hours. ${ }^{6}$ Elevated cortisol levels generally get down to the basic levels by feedback inhibition mechanisms initiated and managed mainly by the hippocampus. In short-term stress, cortisol attaches to the glucocorticoid receptor (GR) and plays antiinflammatory role. ${ }^{6}$ The anti-inflammatory effect of cortisol is ascribed to the suppression of pro-inflammatory genes through signal transduction by the GR. ${ }^{7}$

Although short-term stress works to maintain bodily hemostasis, chronic stress, described as prolonged or exaggerated bodily responses to stressful situations that persists for several hours, days, weeks, months, or years may be harmful. ${ }^{6,8}$ When stressful events are recurring, the cortisol levels are kept at high levels over a long period, damaging hippocampal, where the feedback inhibition begins. ${ }^{9}$ Consequently, even when stressful events end, circulating cortisol levels are stayed at high levels. It has been proposed that the prolonged elevation in cortisol leads to increased GR resistance to cortisol and alternatively increase its affinity for the mineralocorticoid receptor (MR). When attached to the $\mathrm{MR}$, cortisol has pro-inflammatory effects instead, increasing the release of pro-inflammatory factors such as C-reactive protein (CRP). ${ }^{6} \mathrm{CRP}$ is the most widely used proinflammatory biomarker in clinical practice and has been found to be associated with a variety of chronic illnesses such as major depression, coronary artery diseases, cancer, and diabetes mellitus. ${ }^{10,11}$

It has been reported that when dealing with stress, nursing students mostly use inefficient, harmful coping strategies such as avoidance and smoking. These methods have shortlived benefits but are harmful in the long run, resulting in a variety of physical and psychological health problems. ${ }^{12,13}$ Therefore, nursing students may need to use more effective coping strategies. There are several potentially beneficial stress-reduction methods which have been found to help students worldwide cope effectively with stress and its adverse health consequences. ${ }^{14}$ Mind-body therapies are being more widely used worldwide, as they are easily accessible, easy to learn, and inexpensive. ${ }^{15}$ Specifically, mindfulness meditation is a mind-body therapy that has been gaining increasing attention in the recent literature and has demonstrated promising results across different populations and settings. ${ }^{16,17}$ The target groups for mindfulness meditation include patients with psychiatric health problems (eg, depression, anxiety, stress, prosocial behaviors, addiction, and psychosis) and physical health problems (eg, pain, hypertension, obesity, sleep problems, and cancer-related symptoms). ${ }^{16}$ In addition, mindfulness meditation has been used among non-clinical populations, including students, therapists, and prisoners. ${ }^{18}$ Whilst mindfulness meditation has been shown by a growing body of evidence to improve several health and psychological well-being measures in university students, its mechanism of action and how it improves stress and its related health responses remain debated in the literature.

Garland et al proposed a hypothetical causal model that argues for the role of mindfulness meditation in positive reappraisal coping, explaining that attending repeatedly in a mindful way evokes a transitory metacognitive state of mindfulness that remains for as long as that form of attention is sustained. With repeated practices, individuals, including students, can cultivate their predisposition to be mindful in daily life, which is known as trait mindfulness. ${ }^{19}$ Trait mindfulness is described as the cognitive ability to nonjudgmentally observe sensations, thoughts, emotions, and the environment while simultaneously encouraging openness, curiosity, and acceptance. ${ }^{20}$ Previous studies among university students have supported this theoretical claim. A recent randomized controlled trial showed that daily 10-minute sessions of mindfulness meditation for four weeks improved trait mindfulness among university students and staff $(\mathrm{N}=561),{ }^{21}$ and another recent RCT showed that three daily 30-minute sessions of mindfulness meditation significantly improved state mindfulness among university students $(\mathrm{N}=40){ }^{22}$

Based on the mindful coping model, ${ }^{19}$ positive reappraisal is a cognitive coping strategy that enables people to adapt successfully to perceived stress, which is defined as the degree to which events are appraised as stressful and uncontrollable. $^{23}$ For example, a student who perceives given events or situations, such as an exam, as stressful may practice mindfulness meditation to induce an adaptive response by decentering from this stress appraisal into a mode of mindfulness. In turn, this mode allows the student to pay attention to the dynamic process of consciousness themselves rather than their contents, which enhances attentional flexibility and raises awareness. Based on this broadened, metacognitive awareness, the student can then reappraise the given events in a positive way by giving it a new meaning, which may evolve either through a conscious process of reflection or a more automatic process. ${ }^{19}$ The reappraisal of the events then leads to positive emotions such as compassion, trust, and 
confidence, decreasing amygdala reactivity to threatening stressful stimuli. Decreased amygdala reactivity associated with activation of parasympathetic nervous system decreases cortisol levels, which is its high prolonged levels can cause an increase in GR resistance to cortisol and increased circulating markers of inflammation, such as $\mathrm{CRP}^{24}$

Previous studies have also supported the claims of the mindful coping model regarding the effects of mindfulness meditation on perceived stress among university students. A previous RCT showed that eight weekly 75-90-minute sessions of mindfulness meditation reduced study-related stress among university students. ${ }^{25}$ In addition to longterm interventions, RCTs using brief mindfulness meditation, with sessions ranging between 3 daily sessions to 4 weekly sessions, showed that these brief mindfulness meditation interventions decreased perceived stress among university students. ${ }^{26-29}$

In addition to its psychological effects, mindfulness meditation has been found to improve physiological indices of stress such as CRP and cortisol. ${ }^{30,31}$ It is hypothesized that mindfulness meditation buffers stress reactivity, resulting in decreased cortisol responses and proinflammatory markers such as CRP. In a systematic review of $45 \mathrm{RCTs},{ }^{30}$ the authors identified strong evidence of mindfulness meditation leading to reductions in serum cortisol and C-reactive protein. Similarly, another systematic review of RCTs concluded that mindfulness meditation has been found to reduce proinflammatory cytokines, including CRP, which is associated with the reduction of perceived stress and cortisol. ${ }^{31}$

Although an increasing number of studies have demonstrated the effectiveness of mindfulness meditation in improving wellbeing and reducing stress among those with a medical issue/concern and those without/ healthy such as university students, no study has been conducted in Jordan. Therefore, any generalization of these study findings to other populations of different cultures such as nursing students in Jordan is limited due to these cultures' differing belief systems and self-care practices. Also, the number and duration of mindfulness meditation sessions of these previous studies often varied, making it difficult to draw valid conclusion about the effectiveness of mindfulness meditation on stress and proinflammatory responses. Also, there is a lack of evidence related to the effectiveness of mindfulness meditation on stress and its physiological indices in nursing students. Thus, this study aimed to examine the effects of a standardized version of mindfulness meditation on trait mindfulness, perceived stress, serum cortisol, and serum CRP in nursing students.

\section{Materials and Methods Design and Participants}

The current study used a randomized controlled trial of two-group pre-post test blinded design. The study sample included undergraduate nursing students who were conveniently selected from a large university in Jordan and randomly assigned to an experimental and control group. Students were included if they were aged 18 years or over and taking a clinical course. Meanwhile, students were excluded if they were practicing any type of relaxation techniques or taking hypnotics, sedatives, anxiolytics, antidepressants, or anti-hypertensive drugs.

\section{Sample Size Calculation}

$\mathrm{G}^{*}$ Power software 3.1 was used to calculate the required sample size, given a MANOVA test, an alpha of 0.05 , power of 0.95 , number of dependent variables of 4 , number of groups of 2, and effect size of $0.25 .^{22}$ A sample size of 80 was calculated, and an attrition rate of $35 \%$ was added based on a similar previous study. ${ }^{32}$ Therefore, this resulted in a final total sample size of 108 participants.

\section{Instruments}

\section{Socio-Demographic and Behaviors Characteristics}

The participants' socio-demographic and behaviors' characteristics were assessed by questions developed by the study investigators. These characteristics include age, religion, gender, marital status, grade point average (GPA), daily consumption of cigarettes, daily sleeping hours, general health status, nutritional status, coping ability, sleeping quality, and having chronic illness (eg, diabetes mallets, coronary heart diseases, renal failure, cancer, autoimmune inflammatory diseases, mental illnesses, or other). General health status was assessed by the question: "how would you generally rate your overall health?". Nutritional status was assessed by the question: "In general, how would you generally rate your nutritional status?". Coping ability was assessed by the question: "how would you generally rate your coping skills?". Sleeping quality was assessed by the question: "How would you generally rate your sleeping quality?". The last four questions were scored on a Likert scale with $1=$ poor to $5=$ Excellent”. 


\section{Trait Mindfulness}

Trait mindfulness was measured using the Arabic version of the Mindful Attention Awareness Scale (MAAS), ${ }^{33}$ which is a one-dimensional scale that includes 15 items measured on a six-point Likert-type scale ranging from 1 (almost always) to 6 (almost never). The total possible score ranges between 15 and 90, with higher scores indicating higher levels of trait mindfulness. The original scale has been shown to have strong psychometric properties when validated among college students. ${ }^{35}$ Further, the Arabic version of this scale has been shown to have excellent internal consistency and convergent validity for use among Arab populations. ${ }^{33}$ In the current study, the Cronbach's alpha for the Arabic version of the MAAS was 0.79 , indicating good internal consistency.

\section{Perceived Stress}

The Arabic version of The Perceived Stress Scale (PSS) is used to measure the degree to which situations in one's life are appraised as stressful (unpredictable, uncontrollable, and overloaded). ${ }^{36}$ The scale comprises 10 items which are measured on a five-point Likert scale ranging from 0 (never) to 4 (very often). The total possible score ranges from 0 to 40 , with higher scores indicating higher levels of perceived stress. ${ }^{36}$ The original PSS has been validated for use among college students, with an internal consistency coefficient of 0.84 and a test-retest reliability of 0.85 . The Arabic version of the PSS, which was used in the present study, has been shown to have adequate reliability and validity and is considered a suitable instrument for assessing perceived stress among Arab populations. ${ }^{34}$ The exploratory factor analysis revealed two factors with eigenvalues larger than 1.0 ( $45.0 \%$ of variance). The Cronbach's alpha for the overall Arabic scale was 0.8. ${ }^{34}$ In the current study, the Cronbach's alpha for the Arabic version of the PSS was 0.81 , indicating an adequate internal consistency.

\section{Biomedical Markers}

The ELISA method has been found to be accurate, sensitive, specific, and precise in terms of determining the plasma concentration of cortisol. ${ }^{37}$ In the current study, the ELISA protocol, including blood sampling, was strictly followed. Three research assistants with at least 10 years of experience collected $10 \mathrm{~mL}$ blood samples via venipuncture both at baseline and next day morning after the last session, between 8:30-9:30 AM. The results were read using an ELISA reader (Gentaur/GDMS, Kampenhout, Belgium), connected to a computer which showed each reading value. Serum cortisol levels were measured by the following ELISA kit: Cat. Number 33,600 with an intra-assay coefficient of variation of $4.39 \pm 5.92 \%{ }^{22}$ The participants were asked to avoid caffeine consumption on the days of data collection. ${ }^{22}$ To decrease venipuncture-related pain during blood sampling, the EMLA cream was used, as it has been found effective in decreasing venipuncture-related pain among different populations. $^{38}$

\section{Intervention}

In the current study, the $\mathrm{ABC}$ standardized version of mindfulness meditation was used, which includes five 30minute weekly sessions of mindfulness meditation and has been found effective in improving stress and its responses. ${ }^{39}$ An additional 3-hour educational workshop about the intervention was provided before the actual training sessions to enhance the participants' understanding of the intervention. In the initial educational session, the principal investigator (PI) held a Power Point presentation for the participants in each experimental group, which included an explanation of the intervention rationale and procedures and a demonstration of the entire intervention protocol. The PI is an experienced practitioner who received stress-management training at the Psychology Department at Kent State University over 7 years ago and who has, since then, been practicing stressmanagement techniques, especially those used in the current study, on a daily basis. The participants were asked to briefly practice the intervention, and their performance was then evaluated by the PI to make sure that the intervention had been conducted correctly. The PI gave the participants the opportunity to ask questions during the educational session.

During the actual training sessions, the intervention was introduced to the participants in the experimental groups by the PI based on Smith's (2005) guidelines and protocols. ${ }^{39}$ The participants were divided into 6 groups, with 10 to 11 participants in each group. The subgroups met separately in different classrooms, where the interventions were conducted, and provided with identical instructions. Each participant sat in a hard chair behind a desk. The classrooms had tiled floors, and the lights were dimmed during the relaxation phases and turned up when the participants were completing the study questionnaires. A trained research assistant with a master's degree in psychiatric health nursing randomly selected sessions to 
evaluate the delivery of the interventions using checklists produced according to Smith's protocols.

Smith's version of the mindfulness meditation intervention was used in the current study. ${ }^{39}$ The participants were asked to sit upright in a comfortable position, place their feet on the floor, and quietly observe and reflect on internal and external stimuli such as breathing, thought, feeling, physical sensation, and sound, without reactions, judgments, or evaluations. They were asked to quietly attend to, note, and let go of all internal and external stimuli. They were reminded that they should not try to think about, push away, or do anything with these stimuli, and that they should not focus on figuring out the connections between the stimuli. They were asked to simply let each stimulus come and go and wait for the next stimulus. Finally, the participants were told that they should not concern themselves with distractions and that if faced with a distraction, they should note it as yet another passing stimulus (eg, "Ah, a distraction ... how interesting"). ${ }^{39}$

The control group was divided into five subgroups including 10 to 11 participants in each one. The participants were instructed to sit with their eyes closed during the intervention sessions to control for the nonspecific effects of trainer interaction, social interaction, attention, environment, time, and closed eyes. ${ }^{40}$ The timings of the control group sessions were similar to those of the experimental groups, whereby if a given experimental group intervention lasted for 30 minutes, the control group participants would be asked to sit with their eyes closed for 30 minutes also.

\section{Procedure}

After obtaining permission from the dean of the nursing college at Jordan University of Science and Technology (JUST) and the students' instructors, the PI visited the students in their classrooms. After the students' lecture had finished, the PI made an announcement explaining the study title and objectives and asked any students who were interested in participating to contact him either on the phone number assigned for the purposes of the study or via email. The PI arranged an initial meeting to meet with students who were interested in participating, which was to be held on another day in a private, quiet, comfortable, and spacious room at the university. At this meeting, the PI informed the students of the study objectives, risks, and benefits, in addition to answering the students' questions and assuring them that all collected data would be kept confidential. The students were also assured that they had the full right to refuse or discontinue participation at any time and that such refusal or discontinuation would not affect their academic achievement in any way. At the same meeting, informed consent was obtained from participants who agreed to participate and who met the eligibility criteria. After that, the participants were randomly assigned equally to the study groups using a computerized random numbers procedure carried out by a research assistant who was not involved in any other parts of the study. Finally, the dates and times of the sessions were determined.

In the three-hour educational session, baseline measurements of the study variables were taken. Then, the PI delivered a PowerPoint presentation for the participants in each subgroup, as previously explained. At the end of the workshop, to avoid experimental contamination, the participants in each experimental group were asked not to share any information related to the intervention with the participants in the control group. At the end of the intervention (ie, In the next morning after the fifth session), the second measurements of the study variables were taken.

Various strategies were followed to decrease measurement errors that could potentially be affected by variations in the data collection procedure. For example, filling out the questionnaire may be stressful for some subjects, hence influencing the objective measures. Therefore, the selfreport measures were completed after the physical measures were taken. Also, all of the study measures in the intervention and control groups were taken in similar conditions, including similar room temperature and environment. In addition, quiet environments were maintained, with a "Do Not Disturb" sign placed on the doors of the rooms during the interventions and data collection. The data (questionnaire and blood samples) were collected by three research assistants with at least 10 years nursing experiences who were not involved in any other parts of the study.

\section{Human Ethics Statement}

The study was conducted in accordance with the guidelines of the Declaration of Helsinki and approved by the Institutional Review Board at JUST (Reference number: 5/ 121/2019. Date 28.2.2019). Informed consent was obtained from all participants. The study protocol was registered in ClinicalTrail.gov, identifier: NCT05099224.

\section{Statistical Analysis}

Statistical analysis was conducted using SPSS software (version 25). Descriptive statistics were used to describe the sample. The sample and study variables were described by measures of central tendency and dispersion 
appropriate to the level of measurement. Initial independent $t$-tests and Chi-squared tests were conducted to ensure that the randomization across the covariates was successful.

One-way MANOVA was used to test the study hypotheses. However, prior to conducting the MANOVA, univariate and multivariate outliers were examined. Univariate outliers were assessed and appropriately managed if the $z$-scores were larger than 3 or less than -3 for the outcomes within any group. Multivariate outliers were assessed by the Mahalanobis distance test. Univariate normality was assessed by the skewness index of the outcomes for each group. Any skewness index greater than 1 or less than -1 indicates non-normal distribution. The correlations between all of the outcomes using the correlation matrix in order to confirm the MANOVA assumption that the outcomes should not be highly correlated with each other.

Post hoc one-way ANOVAs were run to assess if there were significant differences in each of the outcomes between the study groups. Due to multiple testing, the $\mathrm{p}$-value was adjusted by dividing the $\mathrm{p}$ value of 0.05 by 4 (ie, the number of dependent variables), with a level of 0.0125 considered as the level of significance for the ANOVA tests.

\section{Results}

\section{Attrition}

Of the 305 students who were contacted, 156 agreed to participate and met eligibility criteria. Of those students, 44 did not complete the baseline measurements because of their busy schedule and fear of blood sampling. The remaining 112 students were equally randomized to the experimental group $(n=56)$ receiving mindfulness meditation and the control group $(n=56)$ sitting quiet during mindfulness meditation sessions. Of those students, two students from each group dropped out during intervention and did not complete post-tests because of their busy schedules as well (See Figure 1). The students who dropped out were not statistically different from those who completed the study.

\section{Participant's Characteristics Experimental Group}

In the experimental group $(n=54)$, the mean age of the participants was 19.67 years $(\mathrm{SD}=0.99)$. Most of the participants were single $(n=53)$, unemployed $(n=48)$, and
Muslim $(n=54)$. Among the 54 participants in the experimental group, 18 were male, and 36 were female. On average, the participants rated their general health status as 2.11 out of 5 and their nutritional status as 2.31 out of 5 , whilst the average number of daily sleeping hours was 7.4 hours $(\mathrm{SD}=1.6)$ (See Table 1$)$.

\section{Control Group}

In the control group $(n=54)$, the mean age of the participants was 19.85 (0.76). Similar to the experimental group, the control group included Muslim ( $\mathrm{n}=13,93 \%)$ and Christian $(\mathrm{n}=1,7 \%)$ participants. Further, most of the participants in the control group were single $(n=53)$, unemployed $(n=49)$, and Muslim $(n=54)$. On average, the participants in the control group rated their general health status as. 1.96 out of 5 and their nutritional status as 2.26 out of 5 , whilst the average number of daily sleeping hours was 6.93 hours $(\mathrm{SD}=1.5)$ (see Table 1$)$.

\section{Comparison Between the Groups Based on the Demographic Characteristics and Baseline Scores of the Dependent Variables}

Chi-squared tests and independent t-tests were conducted to investigate any differences between the control and experimental groups based on the selected demographic and health variables. No significant differences were identified between the two groups in terms of gender $\left(\chi^{2}(1)=\right.$ $2.45, \mathrm{p}>0.0125)$, employment status $\left(\chi^{2}(1)=0.10\right.$, $\mathrm{p}>0.0125)$, age $(\mathrm{t}(106)=-0.64, \mathrm{p}>0.0125)$, nutritional status $(\mathrm{t}(106)=0.40, \mathrm{p}>0.0125)$, GPA $\quad(\mathrm{t}(106)=1.30$, $\mathrm{p}>0.0125)$, coping $(\mathrm{t}(106)=-0.32, \mathrm{p}>0.0125)$, or daily sleeping hours $(\mathrm{t}(106)=1.41, \mathrm{p}>0.0125)$ (see Table 1$)$.

Independent t-tests were conducted to examine any pretest mean differences in the dependent variables between the experimental and control groups. The results revealed no significant differences between the groups in terms of the baseline cortisol scores $(\mathrm{t}(106)=0.262$, $\mathrm{p}>0.0125)$, CRP scores ( $\mathrm{t}(106)=-0.949, \mathrm{p}>0.0125)$, baseline perceived stress scores $(\mathrm{t} \quad(106)=0.30$, $\mathrm{p}>0.0125)$, or baseline mindfulness scores $(\mathrm{t}(106)=$ -1.09, $\mathrm{p}>0.0125$ ) (See Table 2).

\section{Comparison Between the Study Groups on Dependent Variables}

One-Way Multivariate Analysis of Variance (MANOVA) tests were run on the four dependent variables: trait 


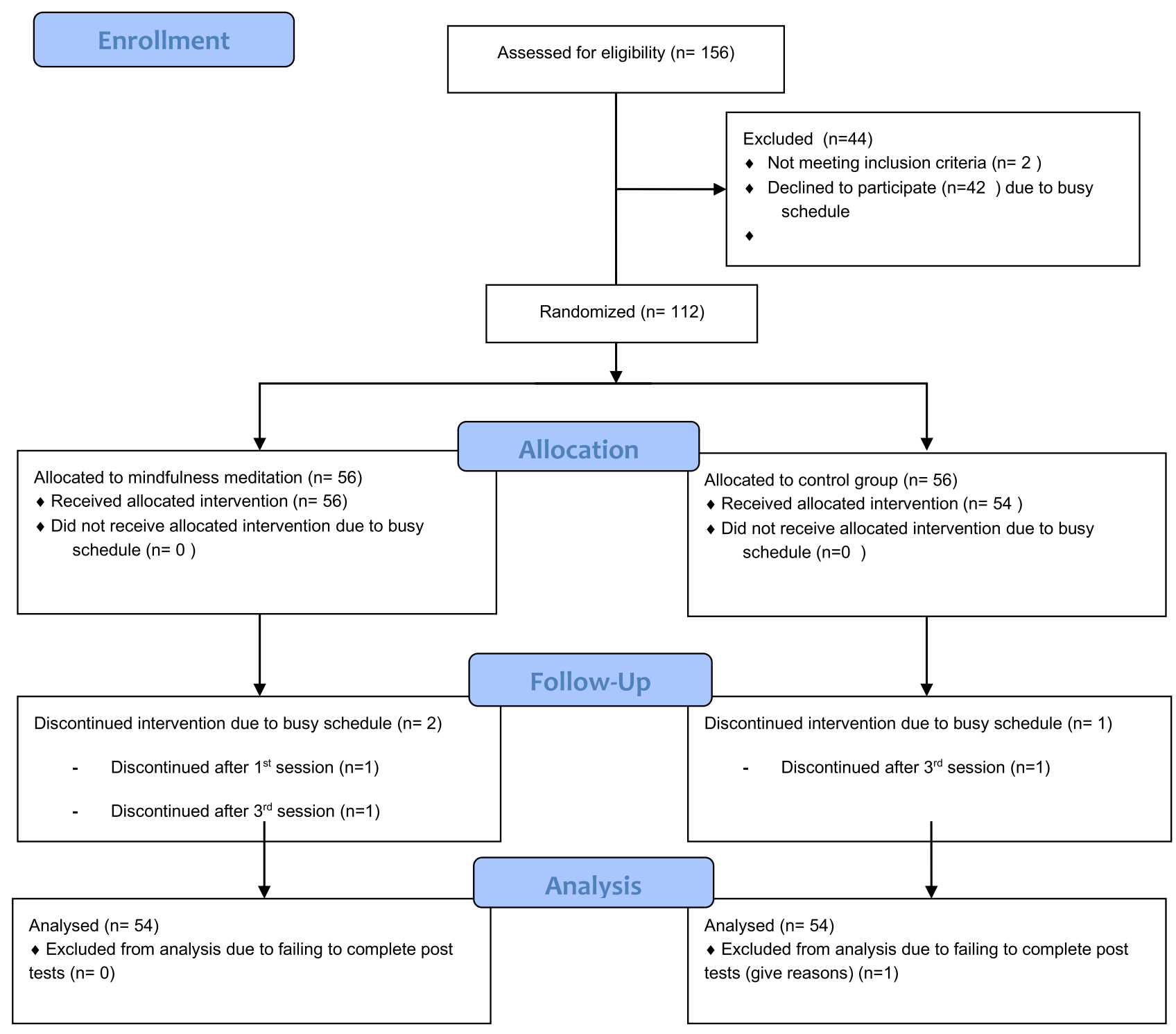

Figure I Study flow diagram for the study participants.

mindfulness, perceived stress, serum cortisol, and serum CRP. The independent variable was group status with two levels: the mindfulness meditation group $(\mathrm{N}=54)$ and the control group $(\mathrm{N}=54)$. No univariate or multivariate outliers were detected. All skewness indices of the dependent variables for each group were within the normal range, indicating the normality assumption was approximately met. The correlation matrix showed no extremely high correlations between any of the dependent variables. Additionally, the Box's $\mathrm{M}$ indicated that the covariance matrices between the groups were equal $(\mathrm{p}=0.492)$.

The results of Levene's F-tests showed that the assumption of homogeneity of variance was met for trait mindfulness, perceived stress, and serum CRP. However,
Levene's $F$-test was statistically significant $(\mathrm{p}>0.05)$ for serum cortisol, indicating a violation of homogeneity of variances. However, Polit (2013) explained that $t$-test is robust against violation of homogeneity of variances when the sample sizes of study groups are approximately equal.

A MANOVA test using PILLAIs' trace as the criterion showed that the study groups were significantly different in term of the combined scores of the four dependent variables (Pillais' Trace $=0.262, \mathrm{~F}(4,103)=9.125, \mathrm{p}<$ $0.001)$. The effect size was 0.26 , suggesting that $26.0 \%$ of the variance in the composite scores of the four dependent variables was accounted for by the study groups.

The results of post hoc comparisons between the study groups showed that two of the one-way ANOVA tests were 
Table I Characteristics of the Sample and Group Comparisons Based on Participants' Characteristics

\begin{tabular}{|c|c|c|c|c|c|c|}
\hline \multirow[t]{2}{*}{ Characteristics } & \multicolumn{2}{|c|}{ n (\%) } & \multicolumn{2}{|c|}{ Mean (SD) } & \multirow[t]{2}{*}{$\chi^{2}(p)$} & \multirow[t]{2}{*}{$t(p)$} \\
\hline & $\begin{array}{c}\text { Experimental } \\
\text { Group }\end{array}$ & $\begin{array}{l}\text { Control } \\
\text { Group }\end{array}$ & $\begin{array}{c}\text { Experimental } \\
\text { Group }\end{array}$ & $\begin{array}{l}\text { Control } \\
\text { Group }\end{array}$ & & \\
\hline Gender & & & & & $2.45(0.11)$ & \\
\hline Female & 36 & 28 & & & & \\
\hline Male & 18 & 26 & & & & \\
\hline Marital status & & & & & $0.00(1.0)$ & \\
\hline Single & 53 & 53 & & & & \\
\hline Married & I & I & & & & \\
\hline Religion & & & & & $0.00(1.0)$ & \\
\hline Muslim & 54 & 54 & & & & \\
\hline Employment & & & & & $0.10(0.75)$ & \\
\hline Yes & $6(7 \%)$ & $5(14 \%)$ & & & & \\
\hline No & 48 (93\%) & 49 (86\%) & & & & \\
\hline Chronic illnesses & & & & & $0.00(1.0)$ & \\
\hline Yes & $0(0 \%)$ & $0(0 \%)$ & & & & \\
\hline No & $54(100 \%)$ & $54(100 \%)$ & & & & \\
\hline Age & & & $19.7(0.99)$ & $19.9(0.76)$ & & $-0.64(0.52)$ \\
\hline Coping & & & $2.20(0.49)$ & $2.24(0.69)$ & & $-0.32(0.75)$ \\
\hline GPA & & & $1.93(0.5 \mid$ & $1.76(0.77)$ & & $1.30(0.76)$ \\
\hline Nutritional status & & & $2.31(0.72)$ & $2.26(0.7 I)$ & & $0.40(0.68)$ \\
\hline Sleep quality & & & $2.33(0.89$ & $2.48(0.84)$ & & $-0.88(0.376)$ \\
\hline Sleeping hours & & & $7.4(1.6)$ & $6.9(1.54)$ & & $1.4 I(0.16)$ \\
\hline $\begin{array}{l}\text { Cigarettes } \\
\text { number }\end{array}$ & & & $2.1(6.2)$ & $5.35(9.7)$ & & $0.62(0.53)$ \\
\hline General health & & & $2.1 I(0.74)$ & $1.96(0.69)$ & & $1.06(0.28)$ \\
\hline
\end{tabular}

Table 2 Baseline Group Comparison Based on the Dependent Variables

\begin{tabular}{|l|c|c|c|c|}
\hline \multirow{2}{*}{ Outcomes } & \multicolumn{2}{|c|}{ Baseline Means (SD) } & \multicolumn{2}{c|}{ t-Tests } \\
\cline { 2 - 5 } & Experimental Group & Control Group & t & 0.262 \\
\hline Cortisol $(\mathrm{pg} / \mathrm{mL})$ & $641.24(234.39)$ & $629.35(236.65)$ & -0.949 & 0.794 \\
\hline CRP $(\mathrm{pg} / \mathrm{mL})$ & $334.95(167.82)$ & $367.89(191.96)$ & 0.30 & 0.76 \\
\hline Perceived Stress & $23.48(3.80)$ & $23.26(3.90)$ & -1.09 & 0.27 \\
\hline Mindfulness & $57.06(17.09)$ & $60.28(13.26)$ & & \multirow{2}{*}{} \\
\hline
\end{tabular}

Abbreviation: CRP, C-reactive protein. 
statistically significant, namely perceived stress $(\mathrm{F}(1)=0.16$, $\mathrm{p}<0.001$, partial $\eta 2=0.08)$ and cortisol $(F(1)=17.10$, $\mathrm{p}<0.001$, partial $\eta 2=0.14)$. Meanwhile, the dependent variables trait mindfulness $(F(1)=9.67, p>0.05$, partial $\eta 2=$ $0.002)$ and CRP $(F(1)=2.58, p>0.05$, partial $\eta 2=0.024)$ were statistically non-significant, although the scores of both the dependent variables improved on the post tests (see Table 3).

\section{Discussion}

The purpose of this study was to examine the effects of mindfulness meditation on serum cortisol levels, serum CRP levels, perceived stress, and mindfulness in nursing students. The results showed that mindfulness meditation was significantly effective in decreasing serum cortisol levels and perceived stress, although the effect size was small. The mindfulness meditation intervention also decreased CRP and increased trait mindfulness, though the results did not reach statistically significant levels.

The mindful coping model explains that repeated mindfulness meditation induces and improves trait mindfulness, described as awareness of cognitive processes. ${ }^{19}$ The results of the current study partially support this theoretical claim with regards to mindfulness meditation decreasing trait mindfulness, although the results were not statistically significant. Previous studies which have used shorter meditation interventions, such as 3-day or 4-week interventions, among university students have reported statistically significant results. ${ }^{21,22}$ The inconsistent results regarding trait mindfulness in the current study may be attributed to that the participants in the current study were considered beginner trainees, as most of them had not practiced mindfulness meditation prior to this study. Therefore, they may need to further practice mindfulness meditation in order to develop higher levels of trait mindfulness and obtain more benefits from this therapy. Kiken et al also found that some people learn to be mindful through mindfulness meditation more readily than others. ${ }^{41}$ Thus, the participants in the aforementioned studies may have been more readily able than the current study participants to learn meditation skills.

Mindfulness meditation has been found to be an effective stress-reduction method that can decrease perceived stress and cortisol levels among university students, ${ }^{27,28}$ which is supported by the findings of the present study. The mindful coping model explains the mechanisms of mindfulness meditation that contribute to decreasing perceived stress and its consequences. ${ }^{19}$ The model posits that students may induce an adaptive response by decentering from the stress appraisal into a mode of mindfulness, enhancing their cognitive skills of attentional flexibility and awareness. Based on this, students can then reappraise a given stressful event in a positive way by giving it new meaning, leading to less autonomic arousal associated with decreased cortisol levels. ${ }^{19}$

As with regards to CRP, the current study results showed that mindfulness meditation non-significantly decreased CRP, which is consistent with previous studies. Bruno et al implemented eight weekly sessions of two hours of mindfulness meditation among medical students and found no significant differences between the control and meditation groups in terms of the inflammatory markers PCR and Tumor Necrosis Factor- $\alpha .{ }^{42}$ Similarly, Cohen et al reported that a mindfulness-based stress reduction method did not significantly decrease CRP among adolescents. ${ }^{43}$ Further, Turner et al found that eight, weekly sessions of mindfulness training lasting 75-90 minutes were unable to buffer the effects of stress on university students' immune system, including CRP. ${ }^{44}$ These non-significant results may be attributed to the small elevation of serum CRP in healthy populations generally, which makes any reduction in serum CRP levels

Table 3 Post-Hoc Univariate Analysis (One-Way ANOVA) for Each Dependent Variable

\begin{tabular}{|c|c|c|c|c|c|c|c|}
\hline \multirow[t]{2}{*}{ Variables } & \multicolumn{2}{|c|}{ Experimental Group } & \multicolumn{2}{|c|}{ Control Group } & \multicolumn{2}{|c|}{ F Tests } & \multirow[t]{2}{*}{ Partial $\eta^{2}$} \\
\hline & $\mathbf{M}$ & SD & $\mathbf{M}$ & SD & $\mathbf{F}$ & $\mathbf{p}$ & \\
\hline Mindfulness & 59.28 & 14.9 & 58.02 & 17.30 & 9.67 & 0.68 & 0.002 \\
\hline Perceived stress & 20.07 & 4.78 & 17.06 & 5.29 & 0.16 & 0.002 & 0.08 \\
\hline Cortisol (pg/mL) & 430.90 & 169.80 & 589.1 & 224.0 & 17.10 & 0.00 & 0.14 \\
\hline CRP $(\mathrm{pg} / \mathrm{mL})$ & 357.49 & 178.11 & 415.3 & 195.5 & 2.58 & 0.11 & 0.024 \\
\hline
\end{tabular}

Abbreviation: CRP, C-reactive protein. 
difficult to notice. In unhealthy populations, such as patients with major depression who may have higher levels of CRP as compared to healthy populations, mindfulness meditation has also been found to be significantly effective in decreasing CRP. ${ }^{45}$

The current study results showed that mindfulness meditation decreased CRP although the results did not reach statistically significant levels. The positive effect of mindfulness meditation on CRP can be explained by that mindfulness meditation employs and develops emotion regulation skills, such as cognitive reappraisal that decrease perceived stress, and the corresponding autonomic arousal. The reduced autonomic arousal is associated with decreased circulating cortisol, consequently reducing resistance of the GR that blocks cortisol binding and its affinity for the mineralocorticoid receptor (MR). As explained previously, these physiological changes result in a reduction in the release of pro-inflammatory factors, such as CRP. ${ }^{24}$

\section{Limitations}

There were key limitations to the current study. Our sample underpowered some statistical tests, which made it difficult to capture subtle effects, especially in terms of CRP and trait mindfulness. Thus, the use of a larger sample size in future studies is recommended. Moreover, although the use of a homogeneous sample from one setting may improve internal validity, it reduces the generalizability of the findings. The current study also used a convenience sampling method, limiting external validity. Thus, it is recommended that future studies recruit samples from multiple settings using probability sampling methods.

\section{Implications}

Despite the study limitations, the current study adds to the body of knowledge on stress and coping. The findings of this study may be used to guide nursing education, nursing practice, and health care policy-making. The positive results regarding the impacts of mindfulness meditation offer a promising tool for nursing students to manage stress and its negative health consequences. Finally, the current study findings may motivate nursing educators to develop and incorporate mindfulness meditation training into nursing curricula.

\section{Conclusions}

The findings of the present study generally support the use of mindfulness meditation as a stress-reduction technique among undergraduate nursing students, as mindfulness meditation was found to significantly improve perceived stress and cortisol. As with regards to trait mindfulness and CRP, improvements were clinically recorded, though they did not reach statistically significant levels. This may be attributed to the use of a short intervention or the use of a convenience sampling method to recruit participants. Therefore, further studies which use longer interventions and probability sampling methods are recommended to further investigate the effects of mindfulness meditation on trait mindfulness and CRP. Mindfulness meditation was found to be a promising tool for nursing students to manage stress and number of health consequences.

\section{Data Sharing Statement}

The data will be available upon a reasonable request from corresponding author Hossam N. Alhawatmeh by email (hnhawatmeh@just.edu.jo).

\section{Acknowledgments}

Thanks to all students who participated in the study. We are also very thankful to the administration of faculty of nursing and nursing educators in Jordan University of Science and Technology. Thanks for Jordan University of Science and Technology for funding of this study and supporting authors.

\section{Disclosure}

The authors declare no conflicts of interest in this work. The funder (Jordan University of Science and Technology) had no role in the design of the study; in the collection, analyses, or interpretation of data; in the writing of the manuscript, or in the decision to publish the results.

\section{References}

1. Foster K, Roche M, Giandinoto JA, et al. Mental health matters: a cross-sectional study of mental health nurses' health-related quality of life and work-related stressors. Int J Ment Health Nurs. 2021;30 (3):624-634. doi:10.1111/inm.12823

2. Al-Zayyat AS, Al-Gamal E. Perceived stress and coping strategies among Jordanian nursing students during clinical practice in psychiatric/mental health courses. Int $J$ Ment Health Nurs. 2014;23 (4):326-335. doi:10.1111/inm.12054

3. Labrague LJ, McEnroe-Petitte DM, Santos DL, et al. Examining stress perceptions and coping strategies among Saudi nursing students: a systematic review. Nurse Educ Today. 2018;65:192-200. doi:10.1016/j.nedt.2018.03.012

4. Chaabane S, Chaabna K, Bhagat S, et al. Perceived stress, stressors, and coping strategies among nursing students in the Middle East and North Africa: an overview of systematic reviews. Syst Rev. 2021;10 (1):1-7. doi:10.1186/s13643-021-01691-9 
5. Kinlein SA, Karatsoreos IN. The hypothalamic-pituitary-adrenal axis as a substrate for stress resilience: interactions with the circadian clock. Front Neuroendocrinol. 2020;56:100819. doi:10.1016/j. yfrne.2019.100819

6. Hannibal KE, Bishop MD. Chronic stress, cortisol dysfunction, and pain: a psychoneuroendocrine rationale for stress management in pain rehabilitation. Phys Ther. 2014;94(12):1816-1825. doi:10.2522/ ptj.20130597

7. Cruz-Topete D, Cidlowski JA. One hormone, two actions: anti-and pro-inflammatory effects of glucocorticoids. Neuroimmunomodulation. 2015;22:20-32. doi:10.1159/000362724

8. Saul AN, Oberyszyn TM, Daugherty C, et al. Chronic stress and susceptibility to skin cancer. $J$ Natl Cancer Inst. 2005;97 (23):1760-1767. doi:10.1093/jnci/dji401

9. Mizoguchi K, Ishige A, Aburada M, Tabira T. Chronic stress attenuates glucocorticoid negative feedback: involvement of the prefrontal cortex and hippocampus. Neuroscience. 2003;119(3):887-897. doi:10.1016/S0306-4522(03)00105-2

10. Knight EL, Jiang Y, Rodriguez-Stanley J, et al. Perceived stress is linked to heightened biomarkers of inflammation via diurnal cortisol in a national sample of adults. Brain Behav Immun. 2021;93:206-213. doi:10.1016/j.bbi.2021.01.015

11. Markozannes G, Koutsioumpa C, Cividini S, et al. Global assessment of C-reactive protein and health-related outcomes: an umbrella review of evidence from observational studies and Mendelian randomization studies. Eur J Epidemiol. 2021;36(1):11-36. doi:10.1007/s10654-02000681-w

12. Khader YS, Alsadi AA. Smoking habits among university students in \{: prevalence and associated factors. East Mediterr Health J. 2008;14 (4):897-904.

13. Khater W, Akhu-Zaheya L, Shaban I. Sources of stress and coping behaviours in clinical practice among baccalaureate nursing students. Int J Humanit Soc Sci. 2014;4(6):194-202.

14. Amanvermez Y, Rahmadiana M, Karyotaki E, et al. Stress management interventions for college students: a systematic review and meta-analysis. Clin Psychol. 2020. doi:10.1111/cpsp.12342

15. Yanyu J, Xi Y, Huiqi T, et al. Meditation-based interventions might be helpful for coping with the Coronavirus disease 2019 (COVID-19). OSF Preprints. 2020. doi:10.31219/osf.io/f3xzq

16. Zhang D, Lee EKP, Mak ECW, et al. Mindfulness-based interventions: an overall review. Br Med Bull. 2021;138(1):41-57. doi: $10.1093 / \mathrm{bmb} / \mathrm{ldab} 005$

17. Virgili M. Mindfulness-based interventions reduce psychological distress in working adults: a meta-analysis of intervention studies. Mindfulness. 2015;6(2):326-337. doi:10.1007/s12671-013-0264-0

18. De Vibe M, Solhaug I, Tyssen R, et al. Mindfulness training for stress management: a randomised controlled study of medical and psychology students. BMC Med Educ. 2013;13(1):107. doi:10.1186/1472-6920-13107

19. Garland E, Gaylord S, Park J. The role of mindfulness in positive reappraisal. Explore. 2009;5(1):37-44. doi:10.1016/j.explore.2008.10.001

20. Kabat-Zinn J. Mindfulness-based interventions in context: past, present, and future. Clin Psychol Sci Pract. 2003;10(2):144-156. doi:10.1093/clipsy/bpg016

21. Lahtinen O, Aaltonen J, Kaakinen J, et al. The effects of app-based mindfulness practice on the well-being of university students and staff. Curr Psychol. 2021. doi:10.1007/s12144-021-01762-z

22. De Sousa GM, de Lima-araújo GL, de Araújo DB, et al. Brief mindfulness-based training and mindfulness trait attenuate psychological stress in university students: a randomized controlled trial. $B M C$ Psychol. 2021;9(1):1-4. doi:10.1186/s40359-021-00520-x

23. Lazarus RS, Folkman S. Stress, Appraisal, and Coping. New York, USA: Springer publishing company; 1984.
24. Pascoe MC, de Manincor M, Tseberja J, et al. Psychobiological mechanisms underlying the mood benefits of meditation: a narrative review. ComprPsychoneuroendocrinology. 2021;6:100037. doi:10.1016/j. cpnec.2021.100037

25. Galante J, Dufour G, Vainre M, et al. A mindfulness-based intervention to increase resilience to stress in university students (the Mindful Student Study): a pragmatic randomised controlled trial. Lancet Public Health. 2018;3(2):e72-e81. doi:10.1016/S2468-2667(17) 30231-1

26. Chiodelli R, Mello LTN, Jesus SN, et al. Effects of a brief mindfulness-based intervention on emotional regulation and levels of mindfulness in senior students. Psicol Reflex Crit. 2018;31:21. doi:10.1186/s41155-018-0099-7

27. Sanada K, Montero-Marin J, Alda Diez M, et al. Effects of mindfulness-based interventions on salivary cortisol in healthy adults: a meta-analytical review. Front Physiol. 2016;7:471. doi:10.3389/fphys.2016.00471

28. Stevens BS, Royal KD, Ferris K, et al. Effect of a mindfulness exercise on stress in veterinary students performing surgery. Vet Surg. 2019;48(3):360-366. doi:10.1111/vsu.13169

29. Tang YY, Tang R, Jiang C, et al. Short-term meditation intervention improves self-regulation and academic performance. J Child Adolesc Behav. 2014;2:4. doi:10.4172/2375-4494.1000154

30. Parsons EM, Dreyer-Oren SE, Magee JC, et al. Evaluating the indirect efects of trait mindfulness facets on state tripartite components through state rumination and state experiential avoidance. J Nerv Ment Dis. 2019;207(6):440-4450. doi:10.1097/ NMD.0000000000000993

31. Heckenberg RA, Eddy P, Kent S, et al. Do workplace-based mindfulness meditation programs improve physiological indices of stress? A systematic review and meta-analysis. $J$ Psychosom Res. 2018;114:62-71. doi:10.1016/j.jpsychores.2018.09.010

32. Bottaccioli AG, Bottaccioli F, Carosella A, Cofini V, Muzi P, Bologna M. Psychoneuroendocrinoimmunology-based meditation (PNEIMED) training reduces salivary cortisol under basal and stressful conditions in healthy university students: results of a randomized controlled study. Explore. 2020;16(3):189-198. doi:10.1016/j. explore.2019.10.006

33. Rayan A, Ahmad M. The psychometric properties of the mindful attention awareness scale among Arab parents of children with autism spectrum disorder. Arch Psychiatr Nurs. 2018;32(3):444-448. doi:10.1016/j.apnu.2018.01.001

34. Almadi T, Cathers I, Mansour AM, et al. An Arabic version of the perceived stress scale: translation and validation study. Int $J$ Nurs Stud. 2012;49(1):84-89. doi:10.1016/j.ijnurstu.2011.07.012

35. Brown KW, Ryan RM. The benefits of being present: mindfulness and its role in psychological well-being. J Pers Soc Psychol. 2003;84 (4):822. doi:10.1037/0022-3514.84.4.822

36. Cohen S, Kamarck T, Mermelstein RA. Global measure of perceived stress. J Health Soc Behav. 1983;24:385-396. doi:10.2307/2136404

37. Levine A, Zagoory-Sharon O, Feldman R, et al. Measuring cortisol in human psychobiological studies. Physiol Behav. 2007;90(1):43-53. doi:10.1016/j.physbeh.2006.08.025

38. Fetzer SJ. Reducing venipuncture and intravenous insertion pain with eutectic mixture of local anesthetic: a meta-analysis. Nurs Res. 2002;51(2):119-124. doi:10.1097/00006199-200203000-00008

39. Smith JC. Relaxation, Meditation, \& Mindfulness: A Mental Health Practitioner's Guide to New and Traditional Approaches. New York, USA: Springer Publishing Company; 2005.

40. Rausch SM, Gramling SE, Auerbach SM. Effects of a single session of large-group meditation and progressive muscle relaxation training on stress reduction, reactivity, and recovery. Int J Stress Manag. 2006;13(3):273-290. doi:10.1037/1072-5245.13.3.273 
41. Kiken LG, Garland EL, Bluth K, et al. From a state to a trait: trajectories of state mindfulness in meditation during intervention predict changes in trait mindfulness. Pers Individ Dif. 2015;81:41-46. doi:10.1016/j.paid.2014.12.044

42. Bruno VHT, Catarucci FM, Habimorad PH, et al. Evaluation of inflammatory markers in the practice of meditation in students of medicine: controlled clinical trial. Psicosom Psiquiatr. 2017; esp:10. ID: biblio-946959.

43. Cohen ZP, Cosgrove KT, Akeman E, et al. The effect of a mindfulness-based stress intervention on neurobiological and symptom measures in adolescents with early life stress: a randomized feasibility study. BMC Complement Med Ther. 2021;21(1):1-4. doi:10.1186/s12906-021-03295-1
44. Turner L, Galante J, Vainre M, et al. Immune dysregulation among students exposed to exam stress and its mitigation by mindfulness training: findings from an exploratory randomised trial. Sci Rep. 2020;10(1):5812. doi:10.1038/s41598-020-62274-7

45. Eisendrath SJ, Gillung E, Hartzler A, et al. Mindfulness-based cognitive therapy associated with decreases in $\mathrm{C}$-reactive protein in major depressive disorder: a pilot study. J Altern Complement Integr Med. 2016;2:010.

\section{Publish your work in this journal}

Advances in Medical Education and Practice is an international, peerreviewed, open access journal that aims to present and publish research on Medical Education covering medical, dental, nursing and allied health care professional education. The journal covers undergraduate education, postgraduate training and continuing medical education including emerging trends and innovative models linking education, research, and health care services. The manuscript management system is completely online and includes a very quick and fair peer-review system. Visit http://www.dovepress.com/testimonials.php to read real quotes from published authors. 